\title{
A pressure-based method for solving low Mach number two-phase flows with surface tension
}

\author{
Takuya YOSHIMOTO* and Junichi OOIDA* \\ * Canon Inc. \\ 3-30-2 Shimomaruko, Ohta-ku, Tokyo, 146-8501, Japan \\ E-mail: yoshimoto.takuya@canon.co.jp
}

Received 18 March 2015

\begin{abstract}
We develop a pressure-based method using a mixture model for solving low Mach number compressible twophase flows which contain surface tension, viscous stress, and heat conduction. We utilize the inviscid mixture model with isobaric-one velocity conditions imposed at the interface, and simply append viscous dissipation and heat conduction, where an isothermal condition is assumed. The continuum surface force model proposed by Brackbill et al. (1992) is used for surface tension effects. The pressure-based, segregated solution procedure is employed, the advection terms are explicitly integrated to suppress the numerical diffusion, and the other terms are implicitly solved by an iterative method. For the stability of the iterative calculation, the mixture sound speed included in the pressure equation is linearized by using the solution of the previous iteration, and the density is directly calculated from the equation of state. The proposed method is validated by conducting a shock tube problem, a one-dimensional oscillating bubble problem, and a two-dimensional static bubble problem governed by the Laplace law. In these problems, the numerical results are in good agreement with exact solutions. Finally, a two-dimensional collapsing bubble problem is presented to demonstrate the applicability of the proposed method.
\end{abstract}

Key words : Pressure-based method, Compressible fluid, Two-phase flows, Low Mach number, Surface tension

\section{Introduction}

Microfluidic devices using growth and collapse of a bubble have been adapted for many industrial applications such as nozzles in ink-jet printers (Asai et al.,1987) and pumps in microchannels (Jun and Kim,1998). To improve the performances of these devices which significantly depend on the bubble behavior, it is important to establish a prediction method for the micro-bubble dynamics. These systems have the following characteristics: the Mach number often becomes low due to high pressure loss in microchannels, and it is difficult to neglect the influence of surface tension because the curvature of the liquid-gas interface becomes significant. Hence, various numerical methods have been proposed to solve compressible two-phase flows with the above characteristics.

In recent years, Can and Prosperetti (2012) and Aanjaneya et al. (2013) proposed a simplified method which treats the liquid as incompressible fluid, with the emphasis on low Mach number flows. In this method, a level set function is used to decide definitely the position of the liquid-gas interface, and the solving equations are altered with the phase. By virtue of this strategy, this method has the advantage that the sound speed of the liquid can be ignored. However, special treatment of the interface is required to prevent the loss of the volume and to keep the appropriate physical quantities. On the other hand, another method is proposed, which is based on the mixture model. In this model, both the liquid and the gas are treated as compressible fluid, and the interface is regarded as the liquid-gas mixture. In addition, the same algorithm is globally applicable, regardless of the phase. Hence, there is no need to take into consideration the difficulties about the interface in this method.

For building methods which utilize the mixture model with surface tension effects, many interesting approaches have been proposed in the literature. Perigaud and Saurel (2005) proposed the five equation model and solved it by hyperbolic 
solver involving capillary effects. Faccanoni et al. (2012) reported a more simple method that is composed of a four equation type model and a finite volume Roe-type solver without a surface tension term. However, in low Mach number flows, these density-based method using explicit time integration are required not only to restrict the time spacing due to the sound speed but also to implement a preconditioning for a stable computation (Braconnier and Nkonga, 2009). Moreover, if the evolution equation of the volume or mass fraction contained in the above mixture models is not casted in a conservative formulation, it is not clear how to evaluate the Rankine-Hugoniot jump condition associated with the construction of the approximate Riemann solver, which preserves thermodynamic consistency.

In contrast, a pressure-based method is able to develop without considering these difficulties and an efficient computation by using a large time spacing can be expected. Yabe and Wang (1991) proposed the CIP-Combined and Unified Procedure (CCUP) method which is a pressure-based segregated solution procedure for the four equation model. The validity of this method for the prediction of the interface dynamics such as sloshing and wave breaking was reported by Himeno et al. (2004), and similar methods were suggested by Xiao (2004) and Kwatra et al. (2009). However, these previous investigations adopt a homogeneous four equation model, and their results are obtained from assuming the phase equilibrium or the localized unphysical mixture model. The latter model involves the Heaviside function uncorrelated with physical quantities in order to estimate the mixture density at the interface region. Because the CCUP method is a powerful numerical tool to simulate practical two-phase flows, it is expected that further improvement is carried out for simulating models which describes the phasic thermodynamic state of the mixture, in order to predict more complex phenomena at the interface. On the other hand, Kapila et al. (2001) derived an isobaric-one velocity five equation model which keeps a thermodynamic consistency of a non-equilibrium seven equation model, and Ooida et al. (2013) reported the $\gamma$-based four equation model reduced from Kapila's model. This five equation model is applied to the underwater explosions using the PISO-like algorithm by Miller et al. (2013), and its good ability for a qualitative prediction is presented. However, they utilized limited equation of state (EOS) such as an isentropic condition to the gas phase and an isothermal condition to the liquid phase, and surface tension effects are not included. In addition, although the CFL number based on the wave speed is less than unity in their numerical examples, it is interesting to confirm the accuracy and the stability of the computation by using high CFL numbers.

The purpose of this paper is to present a mixture model which involves capillary effects on the basis of inviscid four equation model with thermodynamic consistency, and to develop a CCUP-based method for simulating practical low Mach number flows. We utilize a versatile stiffened gas EOS (SG EOS) which is available for water-air two-phase flows. In the proposed method, the advection terms are treated explicitly to suppress the numerical diffusion, and the other terms including the surface tension, the viscous dissipation, the heat conduction, and the acoustic effect are treated implicitly to overcome the severe restriction for the time spacing. To verify the accuracy and the stability of the proposed method, we conducted a one-dimensional shock tube problem, a oscillating bubble problem, and a two-dimensional static bubble problem, and compared the numerical results with the exact solutions. In the second problem, in order to investigate the possibility of efficient computations, we try to take a large CFL number which is larger than unity. Finally, as a more practical problem, a two-dimensional collapsing bubble problem is presented to demonstrate the applicability of the proposed method.

\section{Model}

We adopt the single pressure and velocity four equation model proposed by Ooida et al. (2013) as the governing equations to the Euler system, and straightforwardly append capillary effects. Moreover, we add extra physical effects such as viscous stress and heat conduction in order to improve the applicability to practical problems. The governing equations in this study are summarized as follows,

$$
\begin{aligned}
& \frac{\partial \rho}{\partial t}+\nabla \cdot(\rho \boldsymbol{u})=0, \\
& \frac{\partial \rho \boldsymbol{u}}{\partial t}+\nabla \cdot(\rho \boldsymbol{u} \otimes \boldsymbol{u})+\nabla p=\nabla \cdot \boldsymbol{\tau}+\boldsymbol{F}_{\mathrm{ST}}, \\
& \frac{\partial \rho E}{\partial t}+\nabla \cdot[(\rho E+p) \boldsymbol{u}]=\nabla \cdot(\boldsymbol{\tau} \cdot \boldsymbol{u})-\nabla \cdot \boldsymbol{q}+\boldsymbol{F}_{\mathrm{ST}} \cdot \boldsymbol{u}, \\
& \frac{\partial \Gamma}{\partial t}+\boldsymbol{u} \cdot \nabla \Gamma=\left(\Gamma_{1}-\Gamma_{2}\right)\left(K \nabla \cdot \boldsymbol{u}+\Phi_{1}-\Phi_{2}\right),
\end{aligned}
$$


where $\rho, \boldsymbol{u}, p, \boldsymbol{\tau}, \boldsymbol{F}_{\mathrm{ST}}$, and $\boldsymbol{q}$ denote the density, the velocity, the pressure, the viscous stress tensor, the capillary force, and the heat flux, respectively. The total energy is defined as $E=e+\boldsymbol{u}^{2} / 2$, where $e$ represents the internal energy. The quantity $\Gamma$ and $\Pi$ are given by the mixing rule:

$$
\begin{aligned}
& \Gamma=\sum_{k} \alpha_{k} \Gamma_{k}=\sum_{k} \frac{\alpha_{k}}{\gamma_{k}-1}, \\
& \Pi=\sum_{k} \alpha_{k} \Pi_{k}=\sum_{k} \frac{\alpha_{k} \gamma_{k} \pi_{k}}{\gamma_{k}-1} .
\end{aligned}
$$

Here, $\gamma_{k}$ is the ratio of specific heat, $\pi_{k}$ is a material dependent parameter, and $\alpha_{k}$ is the volume fraction. Subscript $k$ represents species of phases, where $k=1$ and 2 refer to the liquid and the gas, respectively. The mixing rules for the density and the internal energy are defined as:

$$
\begin{aligned}
& \rho=\sum_{k}\left(\alpha_{k} \rho_{k}\right), \\
& \rho e=\sum_{k}\left(\alpha_{k} \rho_{k} e_{k}\right) .
\end{aligned}
$$

We assume that each phase is governed by the SG EOS,

$$
p+\gamma_{k} \pi_{k}=\left(\gamma_{k}-1\right) \rho_{k} e_{k}
$$

and the bulk EOS can be obtained from Eq. (9) and the above mixing rule:

$$
p \Gamma+\Pi=\rho e .
$$

The surface tension effects are described by the Continuum Surface Force (CSF) model (Brackbill et al.,1992):

$$
\begin{aligned}
& \boldsymbol{F}_{\mathrm{ST}}=\sigma \kappa \nabla \alpha_{1}, \\
& \kappa=-\nabla \cdot \frac{\nabla \alpha_{1}}{\left|\nabla \alpha_{1}\right|} .
\end{aligned}
$$

Here, $\sigma$ is the surface tension coefficient and $\kappa$ is the curvature of the interface. Although several options (e.g., the mass fraction) can be selected for evaluating the curvature and the gradient in Eq. (12), we empirically choose the volume fraction. The viscous stress tensor is modeled by effective mixture viscosity,

$$
\boldsymbol{\tau}=\sum_{k} \boldsymbol{\tau}_{k}=\sum_{k} \alpha_{k} \mu_{k}\left(\nabla \boldsymbol{u}+\nabla^{t} \boldsymbol{u}-\frac{2}{3}(\nabla \cdot \boldsymbol{u}) \mathbf{I}\right),
$$

where $\mu_{k}$ is the viscous coefficient of each phase and $\mathbf{I}$ is the unit tensor. The heat flux approximated by Fourier's law and the temperature $T$ are written as:

$$
\begin{aligned}
& \boldsymbol{q}=\sum_{k} \boldsymbol{q}_{k}=-\sum_{k} \alpha_{k} \lambda_{k} \nabla T, \\
& T=\rho e\left(\sum_{k} \alpha_{k} \rho_{k} C_{v, k}\left(\frac{p+\gamma_{k} \pi_{k}}{p+\pi_{k}}\right)\right)^{-1}=\rho e\left(\rho C_{v}\right)^{-1} .
\end{aligned}
$$

Here, $\lambda_{k}$ is the thermal conductivity of each phase, $C_{v}$ is the specific heat capacity at constant volume. Note that we assume the thermal equilibrium which conflicts Kapila's model, where two temperatures are considered for evaluating the heat conduction effects. Note also that an unphysical state occurs in the numerical mixture region when each phase is not able to satisfy the following relation,

$$
\left(\gamma_{1}-1\right) \rho_{2} c_{2}^{2}=\left(\gamma_{2}-1\right) \rho_{1} c_{1}^{2}
$$

where $c_{k}$ is the sound speed of each phase defined as $\rho_{k} c_{k}^{2}=\gamma_{k}\left(p+\pi_{k}\right)$. Nevertheless, we can accomplish an efficient computation without handling the two phasic energy equations by enforcing this assumption. Details of the thermodynamic consistency are summarized in Appendix. The quantity $\Phi_{k}$ related to the viscous stress and the heat conduction is described as:

$$
\Phi_{k}=\left(\sum_{k^{\prime}} \frac{\rho_{k^{\prime}} c_{k^{\prime}}^{2}}{\alpha_{k^{\prime}}}\right)^{-1}\left(\frac{\gamma_{k}-1}{\alpha_{k}}\right)\left(\boldsymbol{\tau}_{k}: \nabla \boldsymbol{u}-\nabla \cdot \boldsymbol{q}_{k}\right) .
$$


The quantity $K$ contained in the right-hand side of Eq. (4) is defined as:

$$
K=\left(\sum_{k} \frac{\rho_{k} c_{k}^{2}}{\alpha_{k}}\right)^{-1}\left(\rho_{2} c_{2}^{2}-\rho_{1} c_{1}^{2}\right)
$$

Regarding the Euler system of the present model, $K \nabla \cdot \boldsymbol{u}$ term of Eq. (4) is a difference between the present model and the $\gamma$-based model proposed by Abgrall (1996) and Shyue (1998). Although this term plays a role to keep the thermodynamic consistency which corresponds to the velocity-mechanical equilibrium, it does not prevent the present model from taking over the oscillation-free property of the $\gamma$-based model for the well-known interface advection problem (Ooida et al., 2013). Furthermore, we note that the present model is similar to Braconnier and Nkonga (2009), except for solving $\Gamma$ instead of $\alpha_{k}$ (note that $\Gamma$ and $\alpha_{k}$ are replaceable by Eq. (5)) and including the irreversible effects in the evolution equation. Finally, we derive the pressure equation by using Eqs. (1)-(6) and (10) as:

$$
\frac{\partial p}{\partial t}+\boldsymbol{u} \cdot \nabla p+\rho c_{\mathrm{w}}^{2} \nabla \cdot \boldsymbol{u}=\Gamma^{-1}\left(\boldsymbol{\tau}: \nabla \boldsymbol{u}-\nabla \cdot \boldsymbol{q}-\left(p\left(\Gamma_{1}-\Gamma_{2}\right)+\left(\Pi_{1}-\Pi_{2}\right)\right)\left(\Phi_{1}-\Phi_{2}\right)\right)
$$

where $c_{\mathrm{w}}$ is defined as $\left(\rho c_{\mathrm{w}}^{2}\right)^{-1}=\Sigma_{k}\left(\alpha_{k} / \rho_{k} c_{k}^{2}\right)$, and is called the Wood sound speed.

\section{Numerical method}

In this study, we utilized the segregated solution procedure which consists of three steps. The first step performs the convection part of the present mixture model, and the second step semi-implicitly treats the viscous stress and the heat conduction. Finally, in the third step, the acoustic effect which involves the capillary force is solved by using an iterative method.

\subsection{Step1: Advection}

In the first step, the advection terms of the governing equations are explicitly time-integrated in order to suppress the numerical diffusion. We adopt the quasi-conservative formulation:

$$
\frac{\phi^{\mathrm{adv}}-\phi^{n}}{\Delta t}+\nabla \cdot\left(\phi^{n} \boldsymbol{u}^{n}\right)-\phi^{n} \nabla \cdot \boldsymbol{u}^{n}=0
$$

where $\phi$ is the vector: $\phi=\left(\boldsymbol{u}, E, \Gamma, p, C_{v}\right)^{t}$, and superscript " $n$ " refers to the previous time step and "adv" indicates the advected value. The general staggered-grid arrangements are used, and the velocity is located at the cell-face and the others are stored at the cell-center. For spatial discretization, the third-order MUSCL interpolation (van Leer, 1979) with the minmod limiter is utilized for the conservative part, and the fourth-order central difference scheme is applied to the velocity divergence of the non-conservative part. The third-order Runge-Kutta scheme (Shu and Osher, 1988) is used for the time-integration. The advected internal energy is evaluated from the total energy and the kinetic energy which is calculated by using the linearly-interpolated velocity. The advected density $\rho^{\text {adv }}$ is calculated from Eq. (10) by using the other advected variables.

\subsection{Step2: Viscous dissipation and heat conduction}

In the second step, we focus on the effects of viscous stress and heat conduction in Eqs. (2)-(4) and (19). First, the viscous forces in the momentum equations are solved with the semi-implicit method proposed by Li et al. (2002). The $x$-component of the semi-discretized equation in two-dimensions is given by,

$$
\rho^{\mathrm{adv}} \frac{u_{x}^{*}-u_{x}^{\mathrm{adv}}}{\Delta t}=\frac{\partial}{\partial x}\left(\mu^{n}\left(\frac{4}{3} \frac{\partial u_{x}^{*}}{\partial x}-\frac{2}{3} \frac{\partial u_{y}^{n}}{\partial y}\right)\right)+\frac{\partial}{\partial y}\left(\mu^{n}\left(\frac{\partial u_{x}^{*}}{\partial y}+\frac{\partial u_{y}^{n}}{\partial x}\right)\right),
$$

and the $y$-component is given in a similar way. Here, the superscript “*” indicates the intermediate value.

Second, the heat conduction terms in the conservation law of the energy are calculated. For the simplicity of computation, we use the evolution equation of the internal energy with the assumptions $\left(\rho C_{v}\right)^{*} \approx \rho^{\text {adv }} C_{v}^{\text {adv }}$ and $\tau_{k}^{*}=\tau_{k}\left(\boldsymbol{u}^{*}, \alpha_{k}^{\text {adv }}\right)$, and solve the system of linear equations for the $T^{*}$ as follows:

$$
\frac{\left(\rho C_{v}\right)^{*} T^{*}-(\rho e)^{\mathrm{adv}}}{\Delta t}=\tau^{*}: \nabla \boldsymbol{u}^{*}+\nabla \cdot\left(\lambda^{\mathrm{adv}} \nabla T^{*}\right) .
$$

In this study, all the linear equation solvers adopt the conjugate gradient method with diagonal scaling. 
Finally, we express the intermediate heat flux and the function related to the dissipation as $\boldsymbol{q}_{k}^{*}=\boldsymbol{q}_{k}\left(T^{*}, \alpha_{k}^{\text {adv }}\right)$ and $\Phi_{k}^{*}=\Phi_{k}\left(\boldsymbol{\tau}_{k}^{*}, \boldsymbol{q}_{k}^{*}, \alpha_{k}^{\text {adv }}, p^{\text {adv }}\right)$, and straightforwardly compute $E^{*}, \Gamma^{*}$, and $p^{*}$ by using $\boldsymbol{u}^{*}, T^{*}$ :

$$
\begin{aligned}
& \rho^{\mathrm{adv}} \frac{E^{*}-E^{\mathrm{adv}}}{\Delta t}=\nabla \cdot\left(\boldsymbol{\tau}^{*} \cdot \boldsymbol{u}^{*}\right)-\nabla \cdot \boldsymbol{q}^{*}, \\
& \frac{\Gamma^{*}-\Gamma^{\mathrm{adv}}}{\Delta t}=\left(\Gamma_{1}-\Gamma_{2}\right)\left(\Phi_{1}^{*}-\Phi_{2}^{*}\right), \\
& \frac{p^{*}-p^{\mathrm{adv}}}{\Delta t}=\left(\Gamma^{\mathrm{adv}}\right)^{-1}\left(\boldsymbol{\tau}^{*}: \nabla \boldsymbol{u}^{*}-\nabla \cdot \boldsymbol{q}^{*}-\left(p^{\mathrm{adv}}\left(\Gamma_{1}-\Gamma_{2}\right)+\left(\Pi_{1}-\Pi_{2}\right)\right)\left(\Phi_{1}^{*}-\Phi_{2}^{*}\right)\right) .
\end{aligned}
$$

\subsection{Step3: Acoustic effects}

In the final step, the acoustic terms which involve surface tension effects are solved implicitly. We adopt the iterative method, and solve the following equations for $p^{m}, \boldsymbol{u}^{m}, E^{m}, \Gamma^{m}$, and $\rho^{m}$ in the outer corrector loop:

$$
\begin{aligned}
& \nabla \cdot \frac{\nabla p^{m}}{\rho^{m-1}}-\frac{p^{m}-p^{*}}{\left(\rho c_{\mathrm{w}}^{2}\right)^{m-1}(\Delta t)^{2}}=\frac{\nabla \cdot \boldsymbol{u}^{*}}{\Delta t}+\nabla \cdot \frac{\boldsymbol{F}_{\mathrm{ST}}^{m-1}}{\rho^{m-1}}, \\
& \frac{\boldsymbol{u}^{m}-\boldsymbol{u}^{*}}{\Delta t}+\frac{1}{\rho^{m-1}}\left(\nabla p^{m}-\boldsymbol{F}_{\mathrm{ST}}^{m-1}\right)=0 \\
& \frac{E^{m}-E^{*}}{\Delta t}+\frac{1}{\rho^{m-1}}\left(\nabla \cdot p^{m} \boldsymbol{u}^{m}-\boldsymbol{F}_{\mathrm{ST}}^{m-1} \cdot \boldsymbol{u}^{m}\right)=0, \\
& \frac{\Gamma^{m}-\Gamma^{*}}{\Delta t}-\left(\Gamma_{1}-\Gamma_{2}\right) K^{m-1} \nabla \cdot \boldsymbol{u}^{m}=0 \\
& \rho^{m} e^{m}\left(\boldsymbol{u}^{m}, E^{m}\right)-\Gamma^{m} p^{m}-\Pi^{m}\left(\Gamma^{m}\right)=0
\end{aligned}
$$

where the superscript " $m$ " denotes the number of iterations. The second-order central difference scheme in spatial discretization and the backward Euler scheme in temporal discretization are utilized. For calculating the pressure equation, we use the inner iteration performed by the linear equation solver. In accordance with the scheme of Himeno et al. (2004), the density $\rho^{m}$ is obtained from Eq. (30) to stabilize the computation by constantly imposing the EOS on the intermediate solutions.

We note that $\rho c^{2}$ in Eq. (26) and $K$ in Eq. (29) are linearized by using the values at the previous outer iteration. Figure 1 shows the sound speed in the liquid-gas mixture which is governed by the following parameters:

$$
\left(\gamma_{1}, \pi_{1}\right)=\left(2.35,10^{6} \mathrm{kPa}\right),\left(\gamma_{2}, \pi_{2}\right)=(1.43,0)
$$

As shown in Fig. 1, the Wood sound speed has a non-monotonic behavior with respect to the volume fraction. Therefore, we linearize these variables to suppress the sensitivity of the variations.

To judge the convergence of the outer corrector loop, we empirically use the normalized $L_{2}$-norm of the residual in two-dimensions,

$$
\left\|R_{j}\right\| \leq \xi\left(\left\|g_{j}\right\| N_{x} N_{y}+\varepsilon\right), \quad \text { for } j=1,2,3,4,
$$

where $R_{j}$ denotes the residual of Eqs. (27)-(30), respectively, $g_{j}$ denotes the scaling parameter $g_{j}=\left(E^{n}, \boldsymbol{u}^{n}, \Gamma^{n}, \rho^{n}\right), N_{x}$ and $N_{y}$ denote the number of cells in each direction, $\varepsilon$ denotes the machine epsilon, and $\xi$ is the criterion which is set to $10^{-9}$ in this paper.

\section{Numerical results}

In this chapter, we provide some numerical examples to illustrate the potential of the proposed method. For all the test cases, the domain is discretized on a Cartesian uniform grid. The time spacing is determined by the CFL condition based on the wave speed. In one-dimensional computation case, the CFL number is defined as the following equation:

$$
\mathrm{CFL}=\max \left(|u|+c_{\mathrm{w}}\right) \frac{\Delta t}{\Delta x} .
$$




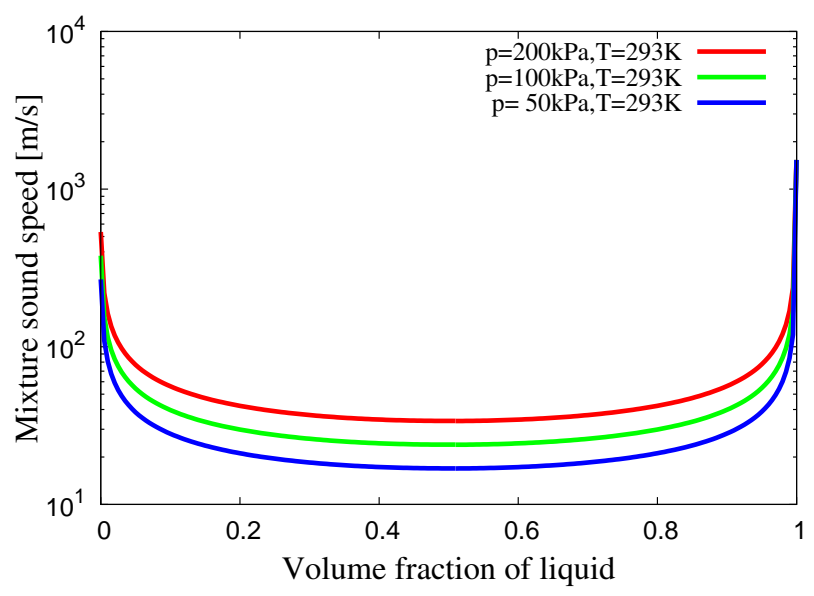

Fig. 1 Sound speed of the liquid water-air mixture.

\subsection{Shock tube problem}

As mentioned above, the present study is focused on low Mach number flows. However, we consider the onedimensional shock tube problem for the two-component perfect gases since it is possible to compare with exact solutions. The problem is solved in the domain for $x \in[-0.5,0.5]$ with 200 cells, and the membrane is positioned at $x=0$. The initial left and right states are,

$$
\begin{array}{ll}
(\rho, u, p, \gamma, \pi)_{L}=\left(1.000,0.0,1.0 \times 10^{5}, 1.4,0.0\right), & \text { if } x<0 \\
(\rho, u, p, \gamma, \pi)_{R}=\left(0.125,0.0,1.0 \times 10^{4}, 1.2,0.0\right), & \text { if } x \geq 0
\end{array}
$$

The surface tension and the dissipations such as viscous and heat transfer effects are ignored, and the CFL number is taken as 0.1 . Figure 2 shows the density and the velocity profiles at $t=5.0 \times 10^{-4}$, which shows good agreement with the exact solutions. In addition, the noticeable oscillations are not observed at the shock front and the contact discontinuity.
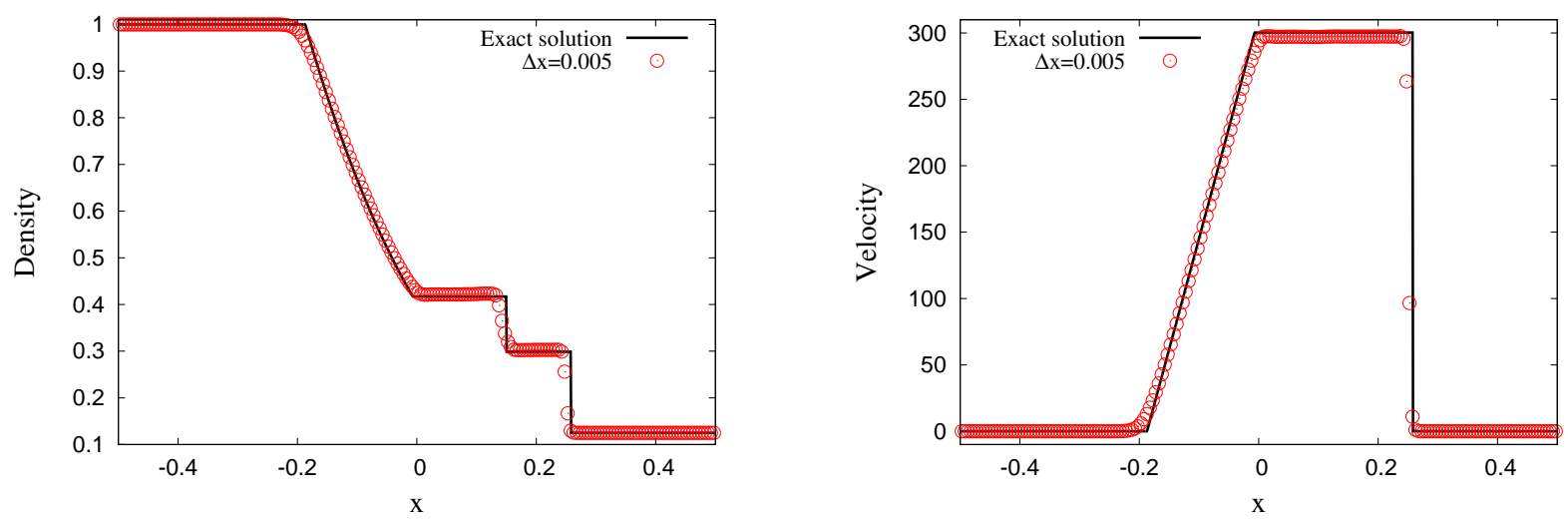

Fig. 2 Profiles of the density and the velocity at $t=5.0 \times 10^{-4}$ for the two-component shock tube problem.

\subsection{Oscillating bubble problem without surface tension}

In this second test case, we consider the one-dimensional oscillating bubble problem. The initial configuration is illustrated in Fig. 3. We consider a $400 \mu \mathrm{m}$ length domain, where each fluid is placed symmetrically. The interfaces of the bubble and the liquid are initially located at $x_{\mathrm{i}}=10 \mu \mathrm{m}$ and $x_{\mathrm{o}}=60 \mu \mathrm{m}$. The initial densities for the liquid phase and the gas phase are $\rho_{1}=2.21933 \mathrm{~g} / \mathrm{cm}^{3}$ and $\rho_{2}=0.00256 \mathrm{~g} / \mathrm{cm}^{3}$, and the initial pressure for the bubble and the other region are set to $500 \mathrm{kPa}$ and $101.3 \mathrm{kPa}$, respectively. The fitting parameters of the EOS are chosen as,

$$
\left(\gamma_{1}, \pi_{1}\right)=\left(4.4,6 \times 10^{5} \mathrm{kPa}\right),\left(\gamma_{2}, \pi_{2}\right)=(1.288,0)
$$

For simplicity, the surface tension and the dissipations are ignored. A fixed pressure boundary condition is used, and the Neumann boundary condition is applied for other variables at outflow boundaries. 
In this problem, we consider a toy model which effectively simplifies this system, and adopt the numerical results of the toy model as "exact" solutions in order to verify the performance of the proposed method. The details of the toy model are presented in Ooida et al. (2013). The maximum Mach number predicted from the toy model is less than 0.1 when the above conditions are applied, and we can regard this test case as a low Mach number flow.

Figure 4 shows the instantaneous result of $K \boldsymbol{\nabla} \cdot \boldsymbol{u}$ term in Eq. (4) which is the difference between the present model and Shyue's model for Euler system, and we can observe that $K \boldsymbol{\nabla} \cdot \boldsymbol{u}$ has an influence on the bubble dynamics at the interface. Figure 5 provides the comparison of the pressure development at $x=0$ and the bubble size for the present model, Shyue's model, and the toy model. The bubble size is defined by the location where $\alpha_{1}=0.5$. As shown in Fig. 5 , the results obtained with the present model are in good agreement with the toy model, irrespective of the grid spacing. In contrast, we can observe errors in the results of Shyue's model. The similar trend with respect to the errors has been reported in Ooida et al. (2013), which solves the same model by the density-based solver. These results obviously indicate the validity of the present model which preserves the thermodynamic consistency.

As stated above, it is interesting to note the maximum possible CFL number for practical problems and the behavior of the solution when large CFL condition is adopted. In Fig. 6, we show the dependence of the pressure development at $x=0$ on the CFL number. The proposed method succeeds in stable computation without spurious oscillations, and the results are in good agreement with the toy model. Note that a slight damping of the pressure occurs at $t \simeq 37 \mu \mathrm{s}$. However, these computations shown in Fig. 6 safely satisfy the convergence criterion. Hence, the damping of the pressure is caused by the numerical diffusion with the increase of the CFL number.

Finally, we investigate the dependence of the total computation time (total CPU time) and the averaged iteration number on $\Delta t$ at $\Delta x=1 \mu \mathrm{m}$, and the results are summarized in Table 1 . The averaged iteration number is defined as the mean value during one period of oscillation. Furthermore, we similarly define the averaged CFL number, and the total CPU time is normalized by the result using the averaged CFL $\simeq 1$ which is almost equivalent to $\Delta t=1 \times 10^{-3} \mu \mathrm{s}$. As shown in Table 1 , when using averaged CFL $\simeq 109$, the normalized total CPU time decreases by approximately $4 \%$ for the case of averaged $\mathrm{CFL} \simeq 1$. It is clear that the proposed method can be expected to simulate efficiently. We note that the normalized total CPU time is not inversely proportional to $\Delta t$, since the variation rate of the averaged iteration numbers tends to increase as the averaged CFL number is increased.

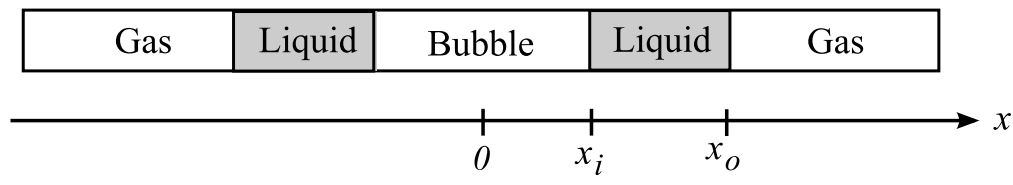

Fig. 3 Configuration of the oscillating bubble problem.

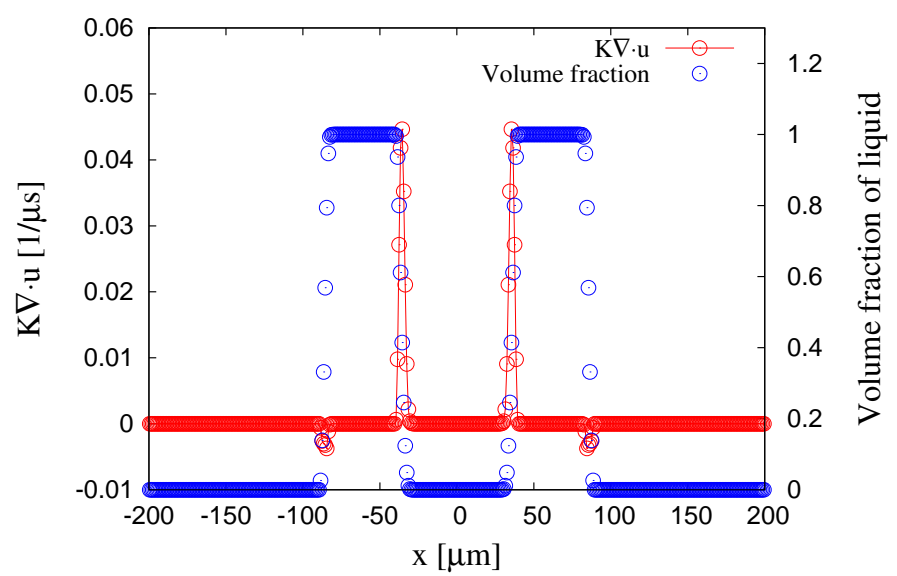

Fig. 4 Spatial profiles of the $K \nabla \cdot u$ and the volume fraction using the present model at $t=5 \mu \mathrm{s}$.

\subsection{Laplace law}

In order to assess the accuracy of surface tension effects, we consider the two-dimensional static bubble governed by the Laplace law. The cylindrical bubble is initially located at the center of a square domain $[-L / 2, L / 2]^{2}$, and the side 

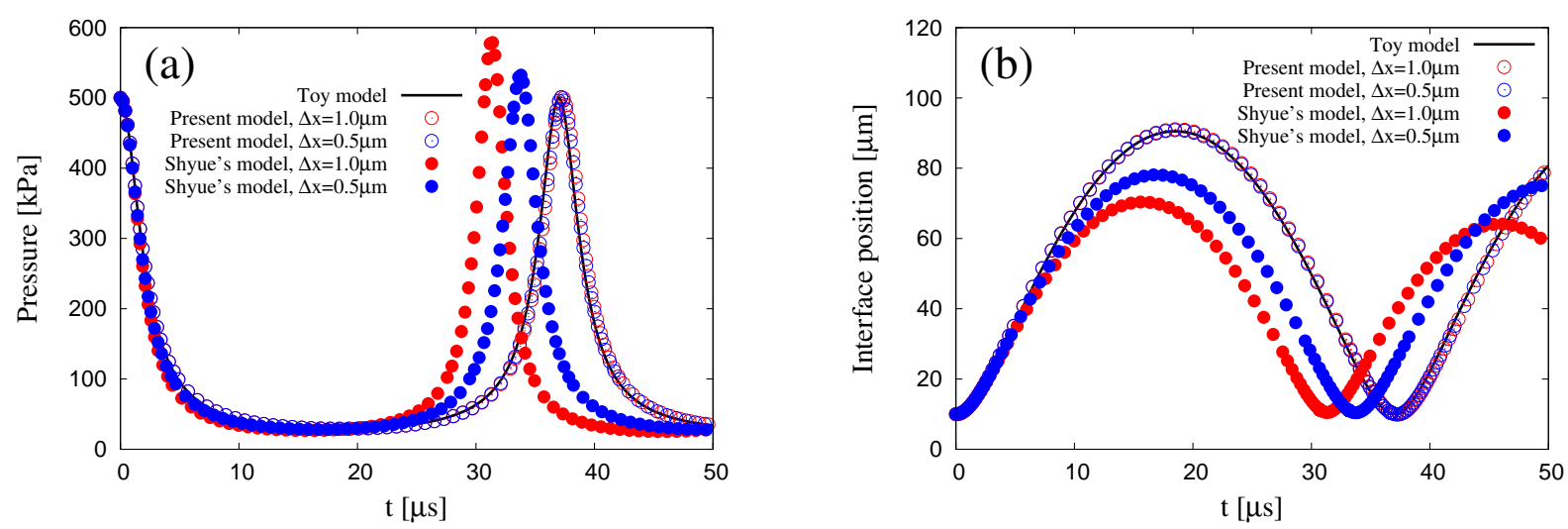

Fig. 5 Comparison of the time histories between the present model(open circles) and Shyue's model(full circles), and the toy model(lines). (a) shows the pressure at the bubble center, (b) shows interface position of the bubble. Red markers and blue markers indicate $\Delta x=1.0,0.5 \mu \mathrm{m}$ respectively. These numerical results are obtained by setting $\mathrm{CFL}=1$.

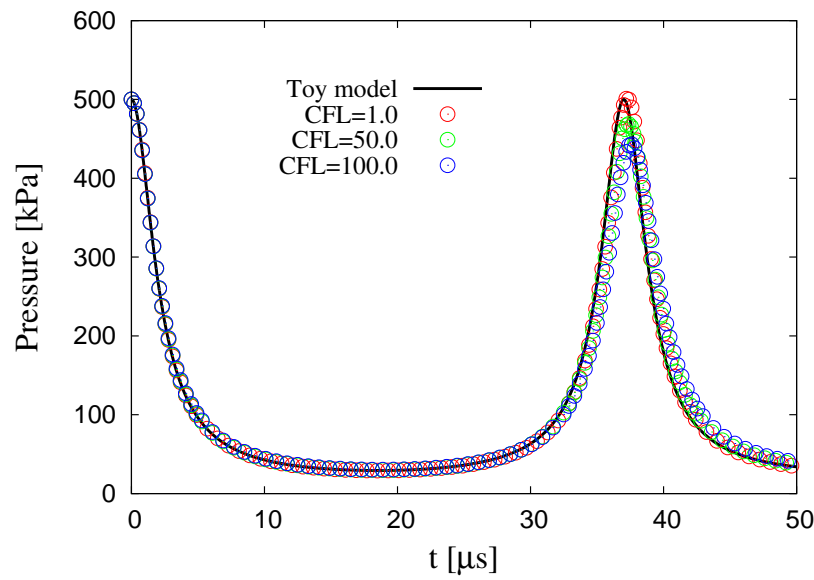

Fig. 6 Dependence of the pressure at the bubble center on the CFL number $(\Delta x=1 \mu \mathrm{m})$.

Table 1 Dependence of the total CPU time and the averaged iteration number on the time spacing.

\begin{tabular}{|c|c|c|c|c|}
\hline \multirow[t]{2}{*}{$\Delta t \times 10^{-3}[\mu \mathrm{s}]$} & \multirow[t]{2}{*}{ Ave. CFL number } & \multirow[t]{2}{*}{ Normalized total CPU time[-] } & \multicolumn{2}{|c|}{$\begin{array}{l}\text { Ave. number } \\
\text { of iterations }\end{array}$} \\
\hline & & & outer & inner \\
\hline 0.5 & 0.548 & 1.959 & 1.0 & 3.1 \\
\hline 1.0 & 1.095 & 1.000 & 1.0 & 5.9 \\
\hline 10.0 & 10.951 & 0.157 & 1.4 & 49.5 \\
\hline 25.0 & 27.376 & 0.094 & 1.9 & 78.9 \\
\hline 50.0 & 54.749 & 0.066 & 2.4 & 108.0 \\
\hline 100.0 & 109.492 & 0.043 & 2.7 & 153.2 \\
\hline
\end{tabular}

length is set to $L=150 \mu \mathrm{m}$ and discretized using 600 cells. The bubble radius is taken as $15 \mu \mathrm{m}$. The EOS parameters are given by using Eq. (31), and the initial conditions are the following:

$$
\begin{aligned}
& \text { Water : } \rho=4.33 \mathrm{~g} / \mathrm{cm}^{3}, p=101.300 \mathrm{kPa} \\
& \text { Bubble : } \rho=7.95 \times 10^{-4} \mathrm{~g} / \mathrm{cm}^{3}, p=106.097 \mathrm{kPa} \text {. }
\end{aligned}
$$

The initial velocity is set to zero. The viscous coefficient of the water and the bubble are set to 2.35 and $0.01 \mathrm{mPa} \cdot \mathrm{s}$, respectively, and the surface tension coefficient is set to $\sigma=71.96 \mathrm{mN} / \mathrm{m}$. The variation of these coefficients is neglected. For simplicity, the heat conduction is ignored. The CFL number is taken as unity.

Figure 7 shows the distribution of the volume fraction $\alpha_{1}$, and Fig. 8 shows the pressure profiles on the x-axis. As can be seen from Figs. 7 and 8, the obtained pressure jump $\Delta p$ across the interface is in good accordance with the Laplace pressure $\Delta p=\sigma \kappa=4.797 \mathrm{kPa}$. Even though parasitic currents are generated near the interface owing to no-special treatment of the CSF model, the interface is maintained the initial shape in this test case. 


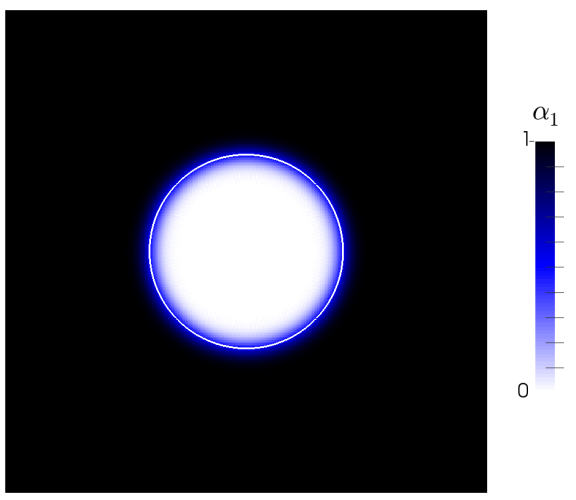

Fig. 7 Distribution of volume fraction $\alpha_{1}$. White line indicates the initial interface.

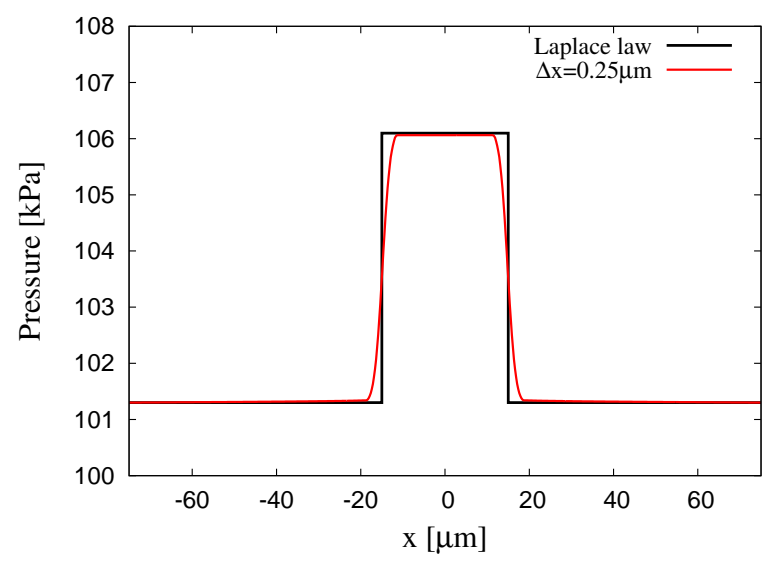

Fig. 8 Pressure profiles as a function of the radius.

\subsection{Collapsing bubble problem}

Finally, we simulate the two-dimensional collapsing of a bubble attached to a solid wall as an example of a more realistic case. In the collapsing process, the re-entrant jet is generated towards the bottom wall, and then the bottom pressure drastically increases by the jet impact, as reported in Plesset and Chapman (1971) and Lauer et al. (2012). In this test case, we try to demonstrate this penetration process by using the proposed method. The configuration of this problem is given in Fig. 9. We assume a water-air system, and use Eq. (31) for the EOS parameters. The initial conditions are,

$$
\begin{aligned}
& \text { Water : } \rho=0.997 \mathrm{~g} / \mathrm{cm}^{3}, p=101.3 \mathrm{kPa}, T=298 \mathrm{~K} \\
& \text { Bubble }: \rho=8.30 \times 10^{-5} \mathrm{~g} / \mathrm{cm}^{3}, p=12.3 \mathrm{kPa}, T=323 \mathrm{~K} .
\end{aligned}
$$

The same conditions as the previous test case are applied to the initial velocity, and to the viscous and the surface tension coefficients. The thermal conductivity of the water and the bubble are set to 600 and $24.1 \mathrm{~W} /(\mathrm{K} \cdot \mathrm{m})$, where the variation is neglected. The fixed pressure boundary condition is utilized for the outlet boundary, and the contact angle at the solid wall is taken to be $90^{\circ}$. Although phase change may affect the collapse speed, we consider that it has a small influence on the qualitative behavior of the collapse such as the jet penetration. Hence, we ignore phase change for simplicity. Moreover, the width of the diffuse interface related to distribution of the physical quantities may also affect the jet intensity. However, we do not investigate the dependence of the interface width because we focus on the validation of the qualitative behavior.

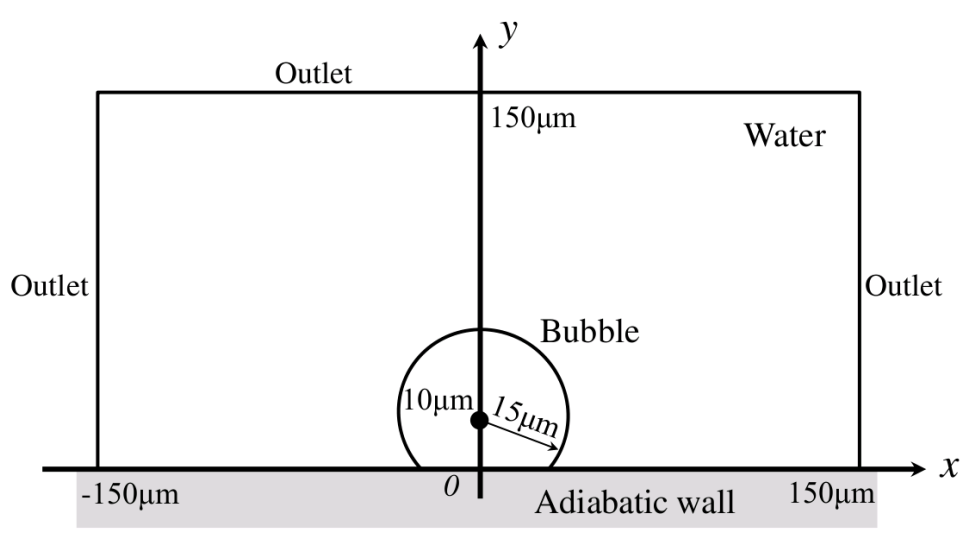

Fig. 9 Configuration of the collapsing bubble problem.

Figure 10 shows the instantaneous distribution of the density, the pressure, and the velocity near the bubble at three selected time instances ( $\mathrm{CFL}=1$, and $\Delta x=\Delta y=0.5 \mu \mathrm{m}$ ). The occurrence of the re-entrant jet and the high pressure region are clearly captured as shown in Fig. 10(a). In addition, it is observed that the re-entrant jet intensely strikes the bottom wall, and that the jet penetration leads to the separation of the bubble with the strong pressure waves (see Fig. 10(b) and (c)). This behavior of the bubble is in agreement with the considered physics, and these results are successfully computed with no spurious oscillations. 
(a) $t=3.6 \mu \mathrm{s}$
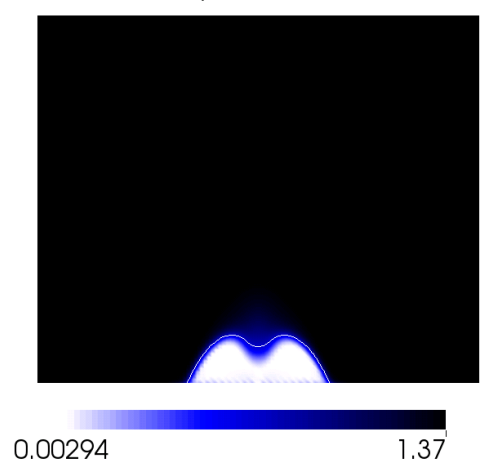

1.37

(b) $t=3.85 \mu \mathrm{s}$

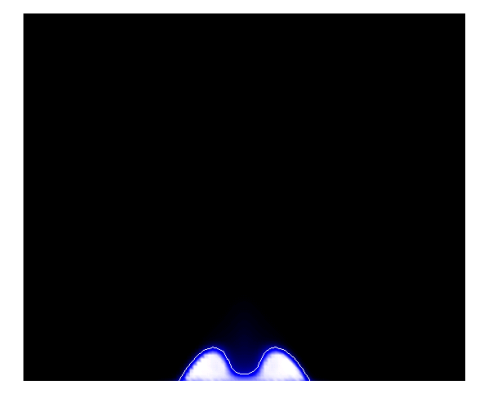

0.0102

1.37

(c) $t=4.1 \mu \mathrm{s}$

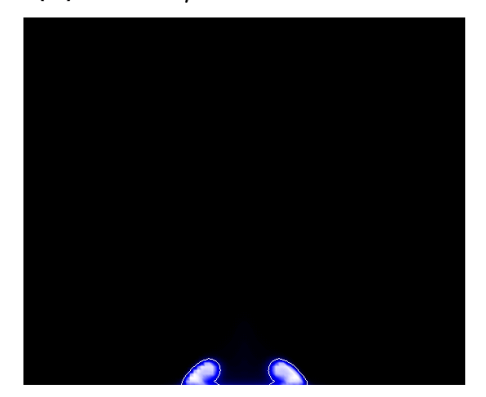

0.0222 1.37
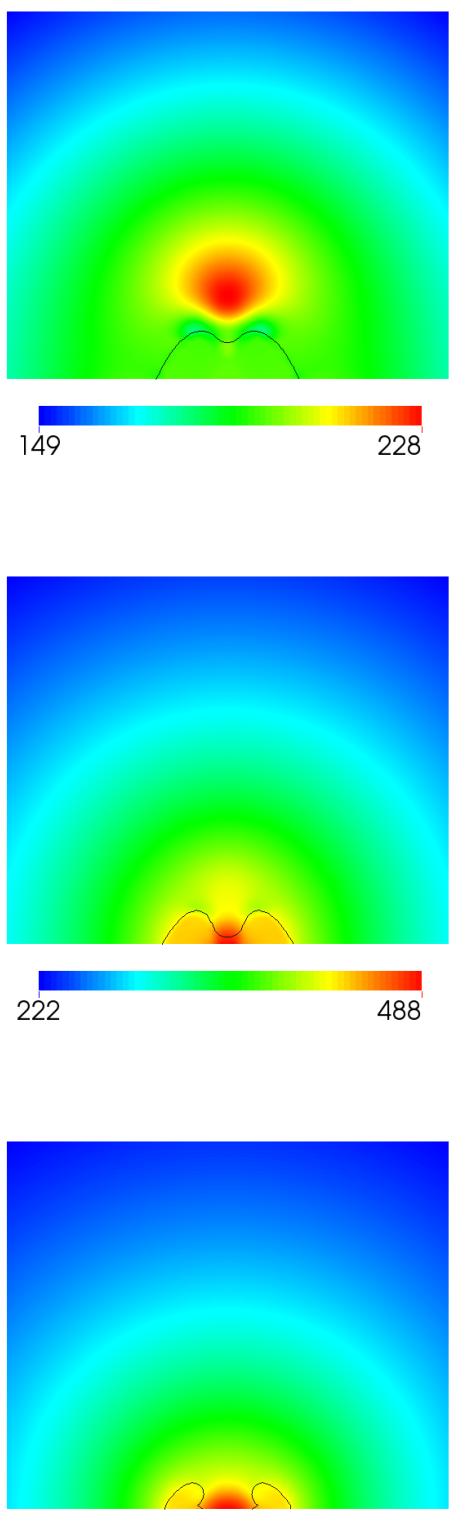

405

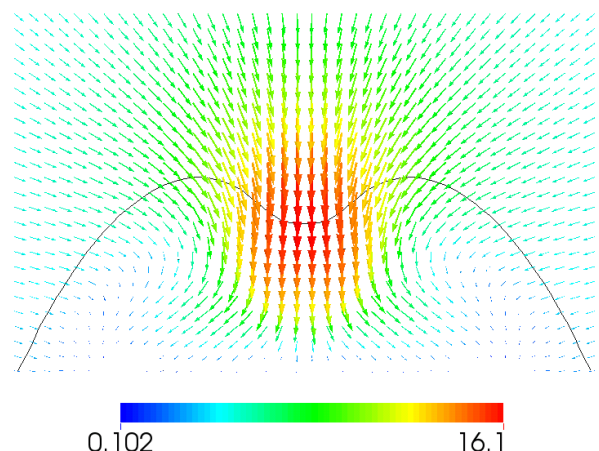

0.102
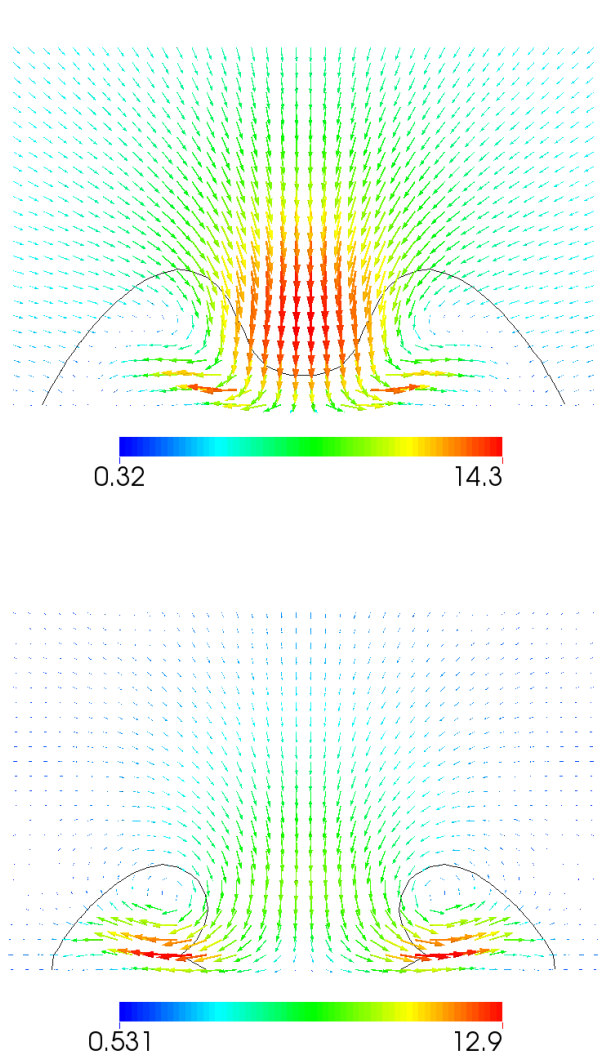

Fig. 10 Time development of flow fields at $t=3.6,3.85$, and $4.1 \mu$ s. The left panels depict the density, the center panels depict the pressure, and the right panels depict the velocity vector. The lines indicate $\alpha=0.5$.

To check the grid convergence, we simulate this system with three different grid spacing $\Delta x=\Delta y=0.25,0.5,1.0 \mu \mathrm{m}$, and the time histories of the bottom pressure at the collapsing position are shown in Fig. 11. It is clear that the influence of the above grid resolution on the pressure evolution is small. Furthermore, we validate the possible CFL number by solving with three different CFL number, and the results are depicted in Fig. 12. It is found that a similar pressure behavior is obtained irrespective of the CFL number. From the obtained results, we expect that the present methodology has the versatility for simulating practical micro-bubble problems.

\section{Conclusions}

We have developed a four equation model, and a pressure-based method for solving low Mach number two-phase flows. The present model is based on thermodynamically consistent, inviscid four equation model and the stiffened gas EOS, and is straightforwardly added the extra physical effects such as surface tension, viscous dissipation, and heat conduction enforcing an isothermal condition. The CCUP method is improved in order to adapt for the present model. We 


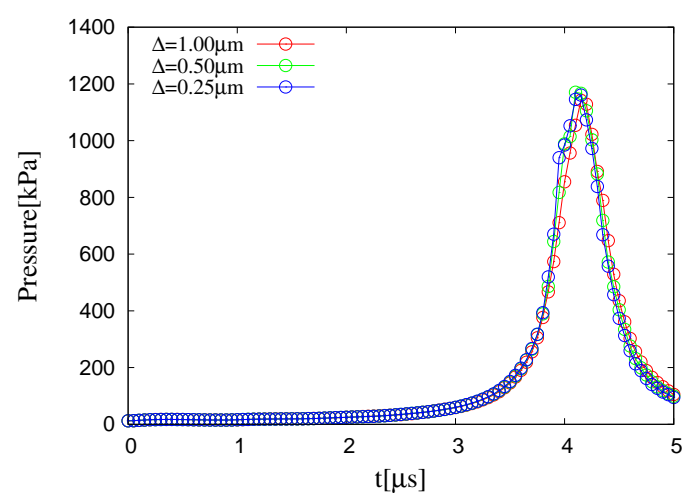

Fig. 11 Dependence of the pressure on the grid spacing at the center of bottom wall $(\mathrm{CFL}=1)$.

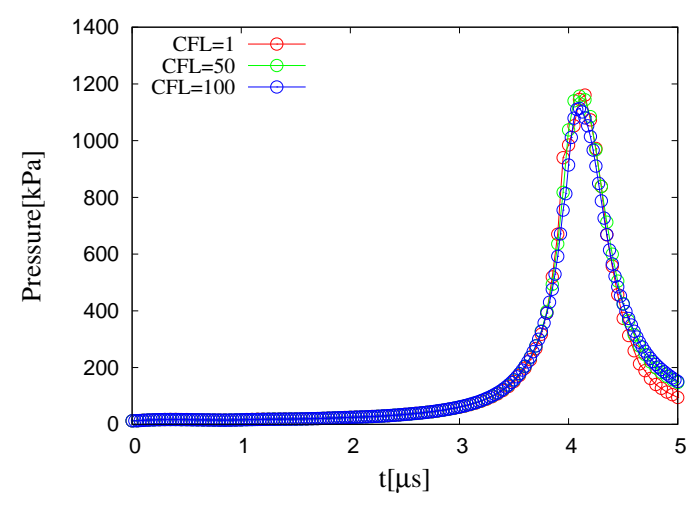

Fig. 12 Dependence of the pressure on the CFL number at the center of bottom wall $(\Delta x=0.25 \mu \mathrm{m})$.

take the non-monotonic behavior of the mixture sound speed into consideration in order to construct an iterative solution procedure for the acoustic part of the model.

A 1-D shock tube problem, a 1-D bubble oscillating problem, and a 2-D static bubble problem are investigated with comparisons to exact solutions, and a 2-D collapsing bubble problem is simulated to assess the versatility of the proposed method. The computations are successively conducted with no obvious oscillations. Moreover, we found that a possible CFL number is more than unity in the dynamic bubble problems. The obtained results suggest that the proposed method has a capability for solving practical micro-bubble flows.

\section{Appendix. Thermodynamic consistency of the present model}

Following Ooida et al. (2013), we obtain the mixture model from a non-equilibrium two-fluid model:

$$
\begin{aligned}
& \partial_{t} \alpha_{k} \rho_{k}+\nabla \cdot \alpha_{k} \rho_{k} \boldsymbol{u}=0, \\
& \partial_{t} \alpha_{k} \rho_{k} \boldsymbol{u}+\nabla \cdot \alpha_{k} \rho_{k} \boldsymbol{u} \otimes \boldsymbol{u}+\nabla \alpha_{k} p=p \nabla \alpha_{k}+\nabla \cdot \boldsymbol{\tau}_{k}, \\
& \alpha_{k} \rho_{k} D_{t} e_{k}=-\alpha_{k} \rho_{k} p D_{t} v_{k}+\boldsymbol{\tau}_{k}: \nabla \boldsymbol{u}-\nabla \cdot \boldsymbol{q}_{k},
\end{aligned}
$$

here, $\partial_{t}=\partial / \partial t, D_{t}=\partial_{t}+\boldsymbol{u} \cdot \nabla$, and $v_{k}$ means inverse of phasic density. We apply the mixing rules to the sum of Eqs. (A.1)-(A.3), and then we can obtain Eqs. (1)-(3) by adding the surface tension to these equations as the external force. Comparing Eq. (A.3) with the thermodynamic relation $D_{t} e_{k}+p D_{t} v_{k}=T_{k} D_{t} s_{k}$, we obtain the evolution equation of the phasic entropy $s_{k}$ :

$$
T_{k} D_{t} s_{k}=\left(\alpha_{k} \rho_{k}\right)^{-1}\left(\boldsymbol{\tau}_{k}: \nabla \boldsymbol{u}-\nabla \cdot \boldsymbol{q}_{k}\right)
$$

In accordance with the scheme of Ooida et al. (2013), substituting Eqs. (A.1) and (A.4) into the pressure equilibrium $p_{1}\left(s_{1}, \rho_{1}\right)=p_{2}\left(s_{2}, \rho_{2}\right)$, the volume fraction equation is obtained:

$$
D_{t} \alpha_{1}=K \nabla \cdot \boldsymbol{u}+\left(\Phi_{1}-\Phi_{2}\right)=\left(\sum_{k} \frac{\rho_{k} c_{k}^{2}}{\alpha_{k}}\right)^{-1}\left(\rho_{2} c_{2}^{2}-\rho_{1} c_{1}^{2}\right) \nabla \cdot \boldsymbol{u}+\left(\Phi_{1}-\Phi_{2}\right) .
$$

By applying the mixing rule Eq. (5) for $\Gamma$, we obtain the evolution equation of $\Gamma$ (Eq. (4)). Because Eq. (A.5) is derived from the pressure equilibrium condition and two-fluid model, it keeps the thermodynamic consistency for the pressure equilibrium. Note that the thermodynamic consistency of the present model is restricted by the thermal equilibrium with respect to the heat conduction.

For simplicity, we ignore the dissipation. We derive the condition which is required to satisfy the thermal equilibrium with using the Wood sound speed, and then the following equation is obtained from two-fluid model:

$$
D_{t} \alpha_{1}=\left(\sum_{k} \frac{\rho_{k} c_{k}^{2}}{\alpha_{k}}\right)^{-1}\left(\gamma_{1} \rho_{2} c_{2}^{2}-\gamma_{2} \rho_{1} c_{1}^{2}\right) \nabla \cdot \boldsymbol{u} .
$$

Therefore, Eq. (16) is the important condition to maintain the consistency of Eq. (A.5) and Eq. (A.6), and to keep the thermodynamic consistency. Although the thermal equilibrium is the severe condition to keep the thermodynamic consistency in the present model, it has the advantage that we can accomplish the efficient computation without high computational cost (i.e. twice implicit calculation for the heat conduction term). 


\section{References}

Aanjaneya, M., Patkar, S. and Fedkiw, R., A monolithic mass tracking formulation for bubbles in incompressible flow, Journal of Computational Physics, 247, (2013), 17-61.

Abgrall, R., How to Prevent Pressure Oscillations in Multicomponent Flow Calculations: A Quasi Conservative Approach, Journal of Computational Physics, 125, (1996), 150-160.

Asai, A., Hara, T. and Endo, I., One-Dimensional Model of Bubble Growth and Liquid Flow in Bubble Jet Printers, Jpn. J. Appl. Phys., 26, (1987), 1794-1801.

Brackbill, J.U., Kothe, D.B. and Zemach, C., A Continuum Method for Modeling Surface Tension, Journal of Computational Physics, 100, (1992), 335-354.

Braconnier, B., and Nkonga, B., An all-speed relaxation scheme for interface flows with surface tension, Journal of Computational Physics, 228, (2009), 5722-5739.

Can, E. and Prosperetti, A., A level set method for vapor bubble dynamics, Journal of Computational Physics, 231, (2012), 1533-1552.

Faccanoni, G., Kokh, S. and Allaire, G., Modelling and simulation of liquid-vapor phase transition in compressible flows based on thermodynamical equilibrium, ESAIM: Mathematical Modelling and Numerical Analysis, 46, (2012), 1029-1054.

Himeno, T., Watanabe, T., Nonaka, S., Naruo, Y. and Inatani, Y., Numerical Analysis of Sloshing and Wave Breaking in a Small Vessel by CIP-LSM, JSME International Journal Series B Fluids and Thermal Engineering, 47, 4, (2004), 709-715.

Jun, T.K. and Kim, C-J., Valveless pumping using traversing vapor bubbles in microchannels, J. Appl. Phys., 83, (1998), 5658.

Kapila, A. K., Menikoff, R., Bdzil, J. B., Son, S. F. and Stewart, D. S., Two-Phase Modeling of DDT in Granular Materials: Reduced Equations, Phys. Fluids, 13, 10, (2001), 3002.

Kwatra, N., Su, J., Grétarsson, J.T., and Fedkiw, R., A method for avoiding the acoustic time step restriction in compressible flow, Journal of Computational Physics, 228, 11, (2009), 4146-4161.

Lauer, E., Hu, X. Y., Hickel, S. and Adams, N.,A., Numerical modelling and investigation of symmetric and asymmetric cavitation bubble dynamics, Computers \& Fluids, 69, (2012), 1-19.

Li, J., Renardy, Y. and Renardy, M., Numerical simulation of breakup of a viscous drop in simple shear flow through a volume-of-fluid method, Phys. Fluids, 12, 2, (2002), 269.

Miller, S.T., Jasak, H., Boger, D.A., Paterson, E.G. and Nedungadi, A., A pressure-based, compressible, two-phase flow finite volume method for underwater explosions, Computers \& Fluids, 87, (2013), 132-143.

Ooida, J., Nakano, K. and Nagane, K., Modified Gamma-Based Model for Solving Low Mach Number Compressible Two Phase Flows, J.Fluid Sci. Tech., 8, (2013), 75-89 .

Perigaud, G. and Saurel, R., A compressible flow model with capillary effects, Journal of Computational Physics, 209, (2005), 139-178.

Plesset, M. S. and Chapman, R. B., Collapse of an Initially Spherical Vapour Cavity in the Neighbourhood of a Solid Boundary, Journal of Fluid Mechanics, 47, 2, (1971), 283-290.

Shu, C.W., and Osher, S., Efficient implementation of essentially non-oscillatory shock-capturing schemes, Journal of Computational Physics, 77, (1988), 439-471.

Shyue, K.M., An Efficient Shock-Capturing Algorithm for Compressible Multicomponent Problems, Journal of Computational Physics, 142, (1998), 208-242.

Van Leer, B., Towards the ultimate conservative difference scheme. V. A second-order sequel to Godunov's method, Journal of Computational Physics, 32, (1979), 101-136.

Xiao, F., Unified formulation for compressible and incompressible flows by using multi-integrated moments I: onedimensional inviscid compressible flow, Journal of Computational Physics, 195, 2, (2004), 629-654.

Yabe, T., and Wang, P., Unified Numerical Procedure for Compressible and Incompressible Fluid, J. Phys. Soc. Jpn., 60, (1991), 2105-2108. 